Bull. Austral. Math. Soc.

57N13, 20F34, 20J05

VOL. $62(2000) \quad[353-356]$

\title{
VIRTUAL SURFACE BUNDLE GROUPS
}

\author{
J.A. Hillman
}

We show that all torsion free groups which are virtual surface bundle groups of type $I$ in Johnson's trichotomy may be realised by aspherical closed smooth 4-manifolds. (This was already known for type II.)

A surface group is the fundamental group of an aspherical closed 2-manifold, and a virtual surface bundle group is a torsion free group which has a subgroup of finite index which is an extension of one surface group by another. If $M$ is a closed 4-manifold with a finite covering space which is the total space of a fibration over a closed 2-manifold then $M$ is aspherical and $\pi_{1}(M)$ is a virtual surface bundle group. The realisation question asks whether every virtual surface bundle group is the fundamental group of some aspherical closed 4-manifold. This question has been studied by Johnson, in a wider context, in a number of papers. (Note that he uses the term Surface to mean closed 2-manifold with negative Euler characteristic. Thus a Surface group is a surface group with trivial centre.)

If $H$ is a subgroup of a group $\pi$ let $C_{\pi}(H), \zeta \pi$ and $\sqrt{\pi}$ denote the centraliser of $H$ in $\pi$, the centre of $\pi$ and the Hirsch-Plotkin radical of $\pi$, respectively. If $K$ is a normal subgroup of $\pi$ conjugation determines a homorphism $\theta$ from $\pi / K$ to the outer automorphism group Out $(K)$. Johnson has shown that if $K$ and $\pi / K$ are surface groups with trivial centre then whether

(I) $\operatorname{Im} \theta$ is infinite and $\operatorname{Ker} \theta \neq 1$;

(II) $\operatorname{Im} \theta$ is finite; or

(III) $\theta$ is injective

depends only on $\pi$ and not on the subgroup $K$. The normal subgroup $K$ is unique if $\pi$ is of type I, and there are at most two such subgroups if $\pi$ is of type II [4].

The trichotomy may be rephrased in terms of centralisers as follows:

(I) $C_{\pi}(K) \neq 1$ and $\left[\pi: K C_{\pi}(K)\right]=\infty$;

(II) $\left[\pi: K C_{\pi}(K)\right]<\infty$; or

(III) $C_{\pi}(K)=1$.

Received 25th October, 1999

Copyright Clearance Centre, Inc. Serial-fee code: 0004-9727/00 \$A2.00+0.00. 
It is easily seen that if $\rho$ is a subgroup of finite index in $\pi$ and $L=K \cap \rho$ then $C_{\rho}(L)=1$ if and only if $C_{\pi}(K)=1$. Since $\rho$ is virtually a product of surface groups if and only if $\pi$ is, commensurate virtual surface bundle groups are of the same type [5]. The trichotomy extends to the cases when $\zeta(\pi / K) \neq 1$; the three types may then be characterised by

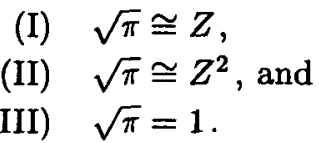

As these properties are shared by commensurate torsion free groups the trichotomy again extends to torsion free groups which are virtually such extensions. On the other hand, it is inappropriate if $\zeta K \neq 1$, as there are then nontrivial extensions with trivial action $(\theta=1)$. Moreover $\operatorname{Out}(K)$ is virtually free and so the action $\theta$ is never injective.

Johnson has shown that all virtual surface bundle groups of type II are realisable [3]. The following theorem shows that all groups of type I are realisable. (Note that we do not assume the centres are trivial).

THEOREM. Let $\pi$ be a torsion free group with normal subgroups $K<G<\pi$ such that $K$ and $G / K$ are surface groups and $[\pi: G]<\infty$. Then $\pi$ is the fundamental group of an aspherical closed smooth 4-manifold which is the total space of a bundle over a 2-dimensional orbifold.

Proof: Let $p: \pi \rightarrow \pi / K$ be the quotient homomorphism. Since $\pi$ is torsion free the preimage in $\pi$ of any finite subgroup of $\pi / K$ is a surface group. As the finite subgroups of $\pi / K$ have order at most $[\pi: G]$, we may assume that $\pi / K$ has no nontrivial finite normal subgroup, and so is the orbifold fundamental group of some 2-dimensional orbifold $B$. Let $F$ be the aspherical closed surface with $\pi_{1}(F) \cong K$. If $\pi / K$ is torsion free then $B$ is a closed aspherical surface, and the result follows from [2]. In general, $B$ is the union of a punctured surface $B_{o}$ with finitely many cone discs and regular neighbourhoods of reflector curves (possibly containing corner points). The latter may be further decomposed as the union of squares with a reflector curve along one side and with at most one corner point, with two such squares meeting along sides adjacent to the reflector curve. These suborbifolds $U_{i}$ (that is, cone discs and squares) are quotients of $D^{2}$ and their orbifold fundamental groups $\pi_{1}^{\text {orb }}\left(U_{i}\right)$ are finite subgroups of $O(2)$. Since $B$ is finitely covered (as an orbifold) by the aspherical surface with fundamental group $G / K$ these finite groups embed in $\pi_{1}^{\text {orb }}(B) \cong \pi / K$, by the Van Kampen Theorem for orbifolds (see [7]).

The action of $\pi / K$ on $K$ determines an action of $\pi_{1}\left(B_{0}\right)$ on $K$ and hence an $F$-bundle over $B_{o}$. Let $H_{i}$ be the preimage in $\pi$ of $\pi_{1}^{\text {orb }}\left(U_{i}\right)$. Then $H_{i}$ is torsion free and $\left[H_{i}: K\right]<\infty$, so $H_{i}$ is a surface group and $\chi\left(H_{i}\right)=\chi(K) /\left[H_{i}: K\right][1]$. Hence $H_{i}$ embeds as a discrete uniform subgroup of $\operatorname{Isom}\left(\mathbb{X}^{2}\right)$, where $\mathbb{X}^{2}=\mathbb{R}^{2}$ if $\chi(K)=0$ and 
$\mathbb{X}^{2}=\mathbb{H}^{2}$ otherwise, and $F$ is a finite covering space of $\mathbb{X}^{2} / H_{i}$. The obvious action of $H_{i}$ on $\mathbb{X}^{2} \times D^{2}$ determines a bundle with general fibre $F$ over the orbifold $U_{i}$.

Since self homeomorphisms of $F$ are determined up to isotopy by the induced outer automorphism class in Out $(K)$, bundles over adjacent suborbifolds have isomorphic restrictions along common edges. Hence these pieces may be assembled to give a bundle with general fibre $F$ over the orbifold $B$, whose total space is an aspherical closed smooth 4-manifold with fundamental group $\pi$, by the Van Kampen Theorem for orbifolds again.

COROLlary. Virtual surface bundle groups of type $I$ are realisable.

Proof: Let $\pi$ be a torsion free group with subgroups $\sigma$ and $N$ such that $[\pi: \sigma]<\infty, N$ is normal in $\sigma$ and $N$ and $\sigma / N$ are surface groups with $\zeta N=$ $\zeta(\sigma / N)=1$. Let $\rho=\cap h \sigma h^{-1}$ and $K=N \cap \rho$. Then $[\pi: \rho]<\infty, \rho$ is normal in $\pi$, and $K$ and $\rho / K$ are surface groups. If $\rho$ is of type $I$ then $K$ is characteristic in $\rho$, by [4], and so is normal in $\pi$. As $\pi / K$ is virtually a surface group, the theorem applies.

Groups of type II need not have such characteristic subgroups. Let $G$ be a surface group with $\zeta G=1$ and let $x$ be a nontrivial element of $G$, and let $\pi=\langle G \times G, t|$ $\left.t\left(g_{1}, g_{2}\right) t^{-1}=\left(x g_{2} x^{-1}, g_{1}\right) \forall\left(g_{1}, g_{2}\right) \in G \times G, t^{2}=(x, x)\right\rangle$. Then $\pi$ is of type II, but has no normal subgroup which is a surface group. A uniform free action of $G$ on $\mathbb{H}^{2}$ determines a uniform free action of $\pi$ on $\mathbb{H}^{2} \times \mathbb{H}^{2}$, by $\left(g_{1}, g_{2}\right) \cdot\left(h_{1}, h_{2}\right)=\left(g_{1}, h_{1}, g_{2}, h_{2}\right)$ and $t .\left(h_{1}, h_{2}\right)=\left(x . h_{2}, h_{1}\right)$, for all $\left(g_{1}, g_{2}\right) \in G \times G$ and $\left(h_{1}, h_{2}\right) \in \mathbb{H}^{2} \times \mathbb{H}^{2}$. The corresponding closed $\mathbb{H}^{2} \times \mathbb{H}^{2}$-manifold is not fibred over a 2-orbifold, although it is doubly covered by the product of two surfaces.

The theorem would also settle the realisation question for groups of type III if it could be shown that every surface bundle group $\tau$ with $\sqrt{\pi}=1$ has a characteristic subgroup $K$ such that $K$ and $\pi / K$ are each surface groups. This is virtually true: if $\pi$ is a surface bundle group and $K$ is a normal surface subgroup such that $\pi / K$ is a surface group with trivial centre then $K$ has only finitely many distinct images $\phi(K)$ under automorphisms of $\pi[6]$. (The key point is that a surface group with trivial centre has only finitely many normal subgroups with a given number of generators).

There is at present no good uniqueness result for normal surface subgroups $K$ with trivial centre when the quotient $\pi / K$ is virtually $Z^{2}$, and it is not known whether every such group $\pi$ is realised by some aspherical closed 4-manifold. (In fact, it also appears to be unknown in how many ways a 3-dimensional mapping torus may fibre over $S^{1}$ ).

On the other hand, if $\pi$ is virtually an extension of a surface group by a normal surface subgroup $K$ with $\zeta K \neq 1$ then either $\sqrt{\pi} \cong Z^{2}$ and $\pi$ is the fundamental group of an aspherical Seifert fibred 4-manifold, with general fibre a torus or Klein bottle (by the theorem), or $\pi$ is virtually poly- $Z$ and is the fundamental group of an 
infrasolvmanifold.

\section{REFERENCES}

[1] B. Eckmann and H. Müller, 'Poincaré duality groups of dimension two', Comment. Math. Helv. 55 (1980), 510-520.

[2] F.E.A. Johnson, 'On the realizability of poly-surface groups', J. Pure. Appl. Algebra 15 (1979), 235-241.

[3] F.E.A. Johnson, 'Extending group actions by finite groups. I', Topology 31 (1992), 407-420.

[4] F.E.A. Johnson, 'A group theoretic analogue of the Parshin-Arakelov rigidity theorem', Arch. Math. (Basel) 63 (1994), 354-361.

[5] F.E.A. Johnson, 'Finite coverings and surface fibrations', J. Pure Appl. Algebra 112 (1996), 41-52.

[6] F.E.A. Johnson, 'A rigidity theorem for group extensions', Archiv Math. (Basel) 73 (1999), 81-89.

[7] G.P. Scott, 'The geometries of 3-manifolds', Bull. London Math. Soc. 15 (1983), 401-487.

School of Mathematics and Statistics

The University of Sydney

Sydney NSW 2006

Australia

e-mail: jonh@maths.usyd.edu.au 